\title{
Materialien für den ägyptischen Übergang zwischen , , i“ und Dentalen
}

Stefan Bojowald

Bonn

\begin{abstract}
In this contribution, the transition between " $i$ " and dentals is considered, which in previous research has not found much attention. The current work is intended to present a representative cross section. Examples will show that, in contrast to common opinion, the transition exists not only since the New Kingdom, but occasionally can be found already in the Old and Middle Kingdom.
\end{abstract}

Keywords: ägyptische Philologie - Übergang zwischen , „i“ und Dentalen.

Der ägyptische Übergang zwischen , , $i^{“}$ und Dentalen ist noch nie in größerem Stil untersucht worden. ${ }^{1}$ Der aufmerksame Beobachter stellt jedoch fest, dass die Fälle für diesen Übergang häufiger als zunächst gedacht sind. In der Forschung sind sie bis auf wenige Ausnahmen konsequent übersehen worden. Die Sicht auf den ganz eigenen

\footnotetext{
${ }^{1}$ Die Auswirkungen dieses Überganges auf die Aphärese von , $i^{\prime \prime}$ vor Dentalen sollen an anderer Stelle abgeklärt werden.
} 
Charakter der Beispiele war daher lange Zeit verstellt, die sich dadurch noch nicht als feste Entität etablieren konnten. Der Übergang könnte aber für all diese Fälle eine wesentlich einfachere Erklärung als die meisten anderen Konzepte bieten. Der vorliegende Beitrag wird sich intensiver mit diesem Phänomen beschäftigen. Die Belege sind mit größtmöglicher Sorgfalt zusammengetragen worden, ohne dass jedoch Anspruch auf Vollständigkeit erhoben werden soll. In Hinblick auf das Alter der Belege sei auf die Angaben in den Klammern verwiesen. Die Tatsache, dass der Übergang entgegen herkömmlichen Meinungen ganz vereinzelt schon im Alten und Mittleren Reich einsetzt, hat dabei für eine kleine Überraschung gesorgt. Die bisher vorherrschende Auffassung war davon ausgegangen, dass er frühestens im Neuen Reich begonnen hat. Die Beispiele sind in den einzelnen Abschnitten nach chronologischen Regeln geordnet.

\section{Der Austausch zwischen , ,i“ und , ,t“}

Im ersten Abschnitt wird der Übergang zwischen , $i^{\text {“ }}$ und,$t$ " breiteren Raum einnehmen. Die Schreibung , $m 3 w i^{\text {‘2 }}$ (NR) für , $m 3 w . t^{\prime \prime}$ „Stab“, die in der Textedition mit der Verlesung des , $t^{\text {“ }}$ - Brotes in die diagonalen ,,$i^{\text {“ } ~ „ S t r i c h e “ ~ e r k l a ̈ r t ~ w o r d e n ~ i s t, ~ k a n n ~ a l t e r n a t i v ~ a u f ~ d i e s e n ~}$ Übergang zurückgeführt werden. Die Schreibung,,$f 3 i^{\text {c*3 }^{3}}$ (NR) für,$f t f t^{\prime *}$ „sich erheben“ ist ebenso zwangsläufig mit diesem Übergang verbunden. Das Wortspiel zwischen , ,il“" „kommen“ und ,itn“" „Scheibe“ in „,i $m$ itn" ${ }^{\prime 4}$ (NR) „als Sonnenscheibe kommen“ setzt ebenfalls notwendiger Weise diesen Übergang voraus, der hier mit dem Austausch zwischen , $i^{\prime \prime}$ und,$n^{\text {“6 }}$ korrespondiert. Die Schreibung

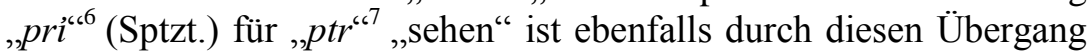

\footnotetext{
${ }^{2}$ Lange, Der Magische Papyrus Harris (1927), 2511.

3 Assmann, Sonnenhymnen in thebanischen Gräbern, Theben 1 (1983), 9.

${ }^{4}$ The Epigraphic Survey in Cooperation with the Department of Antiquities of Egypt, The Tomb of Kheruef Theban Tomb 192, OIP 102 (1980), 30; Zandee, Der Amunhymnus des Papyrus Leiden I 344, verso, Band I (1992), 247; Sandman, Texts from the time of Akhenaten, BibAeg VIII (1938), 4.

${ }^{5}$ Westendorf, Grammatik der medizinischen Texte, Grundriss der Medizin der alten Ägypter VIII (1962), 29.

${ }^{6}$ Jasnow/Zauzich, The ancient Egyptian Book of Thot, A Demotic Discourse on Knowledge and Pendant to the Classical Hermetica, Volume 1: Text (2005), 176. Der von Fecht, Wortakzent und Silbenstruktur, Untersuchungen zur Geschichte der
} 
hergestellt worden. Der Übergang zwischen,$i^{\prime \prime}$ und ,,$t^{\star}$ hilft auch die Schreibung „hmt" ${ }^{8}$ (Sptzt.) für , hmi“" „umstürzen“ besser zu verstehen. Der Übergang hat auch die Verantwortung für das Wortspiel zwischen ,irp“ „Wein“ und „trp“ „Vogel“ in „irp trp "“9 (Sptzt.) „Wein und trp - Vogel“ getragen. Die Schreibung , $t p^{\text {“ } 10}$ (Sptzt.) für „ „ib“ „Herz“ muss hier ebenso notiert werden, die zusätzlich die bekannte Lautverschiebung zwischen , $b^{\text {“ }} \mathrm{zu},, p^{\text {“11 }}$ beinhaltet. Das bereits aus dem Neuen Reich bekannte Wortspiel zwischen , ,il“" „erscheinen“ und ,itn“ „Scheibe“ kehrt mit , ,il itn“"12 (Sptzt.) „Scheibe erscheint“ auch in der Spätzeit wieder.

ägyptischen Sprache, ÄgFo 21 (1960), 108, besprochene Hörfehler zwischen ,ptr“ und ,pri" „, herauskommen“ könnte vielleicht auch mit diesem Austausch begründet werden.

${ }^{7}$ Das Spektrum der Schreibungen für ,ptr“ ist relativ breit gefächert, vgl. , ,p“ bei Cerny, Late Ramesside Letters, BibAeg IX (1939), 66; KRI VI, 211, 10; ,pr’“ “ bei Fecht, Wortakzent und Silbenstruktur, Untersuchungen zur Geschichte der ägyptischen Sprache, ÄgFo 21 (1960), 108, ,pi“ bei Cerny, Late Ramesside Letters, BibAeg IX (1939), 68; Gardiner, Late-Egyptian Stories, BibAeg I (1932), 44a; ,pti“ bei Cerny, Late Ramesside Letters, BibAeg IX (1939), 43; Cerny/Gardiner, Hieratic Ostraca, Volume I (1957), Pl. XXXIV (oPetrie 85 recto, 1/verso 1); Edwards, Hieratic Papyri in the British Museum, Fourth Series: Oracular Amuletic Decrees of the Late New Kingdom, Vol. I, Text (1960), 52, KRI II, 106, 10; KRI II, 721, 10; „pt" bei Jansen-Winkeln, Inschriften der Spätzeit, Teil I: Die 21. Dynastie (2007), 161; ,ptii “ bei Koenig, Le Papyrus Boulaq 6, Transcription, Traduction et Commentaire, BdE 87 (1981), 65; ,pwr“ bei Kákosy/Moussa, SAK 25 (1998), 154.

${ }^{8}$ Burkard, Spätzeitliche Osiris-Liturgien im Corpus der Asasif-Papyri, Übersetzung, Kommentar, Formale und inhaltliche Analyse, ÄAT 31 (1995), 188 n. 75.

${ }^{9}$ Kaplony-Heckel, Land und Leute am Nil, nach demotischen Inschriften, Papyri und Ostraka, Gesammelte Schriften, Teil 2, ÄgAb 71 (2009), 924/935.

${ }^{10}$ de Wit, Les inscriptions du temple d'Opet, a Karnak III, Traduction intégrale des textes rituels - Essai d'interprétation, BibAeg 13 (1968), 135 (308).

${ }_{11}$ Sethe, Das aegyptische Verbum im Altaegyptischen, Neuaegyptischen und Koptischen, Erster Band, Laut- und Stammeslehre (1899), 121; Westendorf, Grammatik der medizinischen Texte, Grundriss der Medizin der alten Ägypter VIII (1962), 23 zum , $p-b$ - $b$ - Ablaut gerade bei der Wurzel „t $p$ “vgl. Fecht, Wortakzent und Silbenstruktur, Untersuchungen zur Geschichte der ägyptischen Sprache, ÄgFo 21 (1960), 104 (§ 194)/109 (§206).

${ }^{12}$ Waitkus, Die Texte in den unteren Krypten des Hathortempels von Dendera, MÄS 47 (1997), 35. 


\section{Der Austausch zwischen , ,i“ und,$t^{6 *}$}

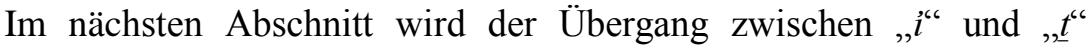
eingehender betrachtet. Der Übergang hat die Voraussetzung für das

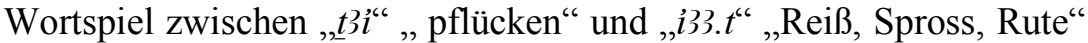

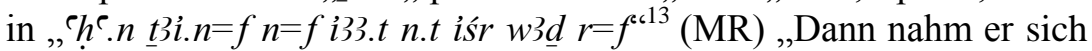
die Rute der Tamariske, die noch ganz grün war." geschaffen. Der Übergang lässt sich auch im Wortspiel zwischen , iri" "machen“ und „triin" „Panzer“ in ,iri triin - Panzer machen“"14 (NR) dingfest machen, bei dem außerdem der schon erwähnte Austausch zwischen ," und , $n^{\prime \prime}$ hinzukommt. In den Wortspielen zwischen ,Imn“ und „mtnn" „Straße/Weg“ in ,'Imn $h r m \underline{t}{ }^{\prime \prime \prime 15}$ (NR) „Amun auf der Straße“ und „Imn p3 nb n3 mtn.w.w'16 (NR) „Amun, Herr der Wege“ lässt sich dieser Übergang in Verbindung mit einer Metathese nachweisen. Der Übergang hat auch bei der Schreibung „śbi“'17 (NR) für ,śbt "lachen“ eine Schlüsselrolle gespielt, die als eines der wenigen Beispiele für diesen Übergang immer schon erkannt worden ist. Das Wortspiel

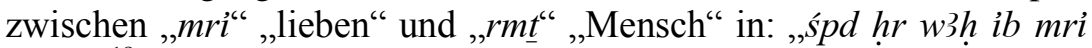
$r m t . w^{{ }^{618}}(\mathrm{NR})$,Der (=Schreiber) umsichtig und aufmerksam ist, der die Menschen liebt" bezieht ebenfalls den Übergang zwischen ,, " $"$ " und ,${ }^{\prime \prime}$ " mit ein. Der Übergang hat sich auch in den Schreibungen ,,$m 3 i^{* 19}$

\footnotetext{
${ }^{13}$ Sethe, Aegyptische Lesestücke zum Gebrauch im akademischen Unterricht, Texte des Mittleren Reiches, Dritte unveränderte Auflage (1959), 20.

${ }^{14}$ Gardiner, Ancient Egyptian Onomastica, Text, Volume I (1947), 68* zum „trỉin” Panzer vgl. auch Albright, The Vocalization of the Egyptian Syllabic Orthography, Neudruck der Ausgabe 1934, American Oriental Series Vol. 5 (1966), 36/65; Helck, Die Beziehungen Ägyptens zu Vorderasien im 3. und 2. Jahrtausend v. Chr., 2., verbesserte Auflage, ÄgAb 5 (1971), 525.

${ }^{15}$ Vleeming, Papyrus Reinhardt, An Egyptian Land List from the Tenth Century B.C., Hieratische Papyri aus den Staatlichen Museen zu Berlin - Preussischer Kulturbesitz, Lieferung II (1993), 18.

${ }^{16}$ Habachi, Kusch 8 (1960), 47.

${ }^{17}$ Erman, Akademieschriften (1880 - 1928), Teil 2: 1911 - 1928 (1986), 524; Caminos, Late-Egyptian Miscellanies, BES I (1954), 325.

${ }^{18}$ Fischer-Elfert, Die Satirische Streitschrift des Papyrus Anastasi I., Übersetzung und Kommentar, ÄgAb 44 (1986), 29/30i. Die Existenz des Übergangs zwischen , „“ und ,$t^{t *}$ widerlegt die These von Derchain-Urtel, in Lüscher, HAT 6 (2000), 44, wonach

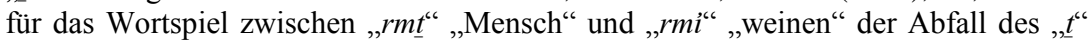
im ersten Wort unabdingbar war.

${ }^{19}$ WB II, 34; Botti/Peet, Le giornale della necropoli di Tebe, Papiri ieratici del Museo di Torino (1928), Tav. 24, recto VIII, 7.
} 


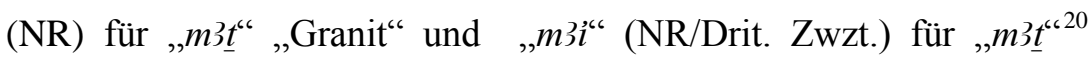
„denken“ artikuliert. In gleicher Weise ist hier das Wortspiel zwischen

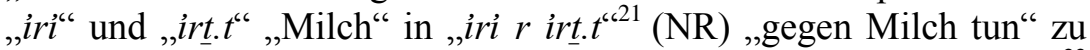

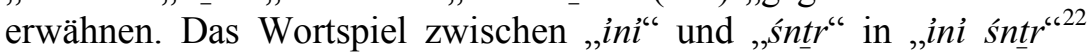
(NR) „Weihrauch bringen“ ist ebenfalls mittels dieses Übergangs gebildet, wobei obendrein die Verschreibung zwischen , , $l^{\text {“6 }} \mathrm{zu},{ }^{\prime \prime 6}$ wegen der Ähnlichkeit der Zeichen im Hieratischen sowie der Lautwandel zwischen , $\iota^{\prime \prime}$ und,$r^{\prime 6}$ eingetreten sind. Die nächsten Beispiele für diesen Übergang können als Einheit verstanden werden, da sie alle drei aus späten Wortspielen zwischen ,iri“، auf der einen Seite und ,irt.t. „Milch“ auf der anderen Seite bestehen. Die Reihe kann mit dem Wortspiel zwischen, ,iri" und ,irt. $t^{* 6}$ in , ,iri $m$ irt. $t^{\text {*623 }}$ (Sptzt.) „durch Milch erhalten“ begonnen werden. Der Übergang ist auch Ausgangspunkt für das Wortspiel zwischen ,,iri ${ }^{“ 6}$ und ,irt.t.t“ „Milch“ in ,iri irt.t. ${ }^{\text {" }}{ }^{24}$ (Sptzt.) „Milch(produktion) garantieren“

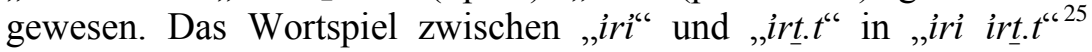
„Kühe bereiten Milch“ geht auf die gleiche Ursache zurück. Das Wortspiel zwischen ,, $3 . t$ " „Hügel“" und ,t $\underline{3}$ mm.t“ in der Ortsbezeichnung ,, $3 . t-\underline{t} 3 m . t^{, 26}$ (Sptzt.) kommt auch nicht ohne diesen Übergang aus. Die nicht ganz sichere Schreibung ,,$\underline{b} b^{627}$ (Sptzt.) für , ,ib3“ „,tanzen“ ließe sich ebenfalls argumentativ mit diesem Übergang begründen. Das Wortspiel zwischen ,iri" „errichten“ und „trrï ““ „Wall“ in: „iri

${ }^{20}$ Gardiner, Ancient Egyptian Onomastica, Text, Volume I (1947), *3; Théodorides, AIP 23 (1979), 97 n. 13; Caminos, A Tale of Woe, From a Hieratic Papyrus in the A.S.Pushkin Museum of Fine Arts in Moscow (1977), 58. Das bei Caminos und Théodorides für eine Schreibung von , $m 3 \underline{t}^{\prime \prime}$,denken“ gehaltene Wort „ $m 3 i^{\prime \prime}$ ist von Allam, JEA 61 (1975), 151, als Schreibung für , $m 3 t^{\prime \prime}$ „Granit“ gedeutet worden.

${ }^{21}$ Erman, Zaubersprüche für Mutter und Kind aus dem Papyrus 3027 des Berliner Museums (1901), 37.

${ }^{22}$ Cerny, Papyrus Hiératiques de Deir el-Médineh, Tome I [ $\left.\mathrm{N}^{\mathrm{os}} \mathrm{I}-\mathrm{XVII}\right]$, DFIFAO 8 (1978), 21 (6)/(7).

${ }^{23}$ Sander-Hansen, Die religiösen Texte auf dem Sarg der Anchnesneferibre (1937), 78.

${ }^{24}$ Breyer, Tanutamani, Die Traumstele und ihr Umfeld, ÄAT 57 (2003), 148.

${ }^{25}$ Junker, Das Geburtshaus des Tempels der Isis in Philä (1965), 343.

${ }^{26}$ Lepsius (Naville/Borchardt Hrsg.; Sethe Bearb.), Denkmäler aus Aegypten und Aethiopien, Text, Dritter Band Theben (1900), 125; Eaton-Krauss/Jansen-Winkeln, MDAIK 57 (2001), 6; Jansen-Winkeln, Inschriften der Spätzeit, Teil I: Die 21. Dynastie (2007), 18/19.

${ }^{27}$ Burkard, Spätzeitliche Osiris-Liturgien im Corpus der Asasif-Papyri, Übersetzung, Kommentar, Formale und inhaltliche Analyse, ÄAT 31 (1995), 51. 
trri $^{\text {“28 }}{ }^{28}$ (Sptzt.) „Wall errichten“ stellt einen weiteren Indikator für diesen Übergang dar. Die Gemeinsamkeiten mit dem oben genannten Wortspiel zwischen ,iri" machen“ und „trïin“ „Panzer“ sind nicht zu übersehen. Das schon aus dem Neuen Reich bekannte Wortspiel zwischen , $m r i$ " und , $r m \underline{t}^{\text {" }}$ (s. o.) ist auch im späten Pflanzennamen

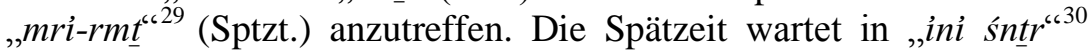
(Sptzt.) „Weihrauch bringen“ ebenfalls mit einem Wortspiel aus ,ini“ „bringen“ und ,śntr" „Weihrauch“" auf, das schon im Neuen Reich begegnet war.

\section{Der Übergang zwischen , ,i“ und , $d^{\text {‘“ }}$}

Im dritten Abschnitt wird der Übergang zwischen , ,i“ und „, $d^{\text {“ }}$ im Vordergrund stehen. Das Wortspiel zwischen ,iri" und „, $d k r^{\prime \prime}$ in: , ,m iri $d k r \quad r=i^{\text {“31 }}$ (MR) ,übe keinen Druck gegen mich aus“ geht unmittelbar auf diesen Übergang zurück. Der hier ebenfalls zu fassende Austausch zwischen, , i“ und Gutturalen soll an anderer Stelle in aller Ausführlichkeit beleuchtet werden. Der Übergang hat auch die Grundlage für das Wortspiel zwischen ,inh“ „umhüllen“ und „dnh

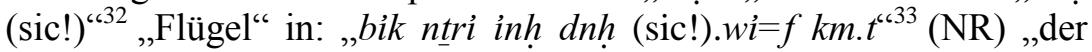
göttliche Falke, dessen beide Flügel Ägypten umhüllen“ gebildet. Die Wirkung des Wortspiels zwischen , $i l h^{\prime \prime}$ „Sumpfpflanze“ und ,idh“" „Delta“ in: „mi ỉh $m$ idh $h^{\text {“34 }}$ (NR), ,wie eine Sumpfpflanze im Delta“ ist ebenfalls diesem Übergang zu verdanken. Der Übergang lässt sich auch als Grund für das Wortspiel zwischen ,iri" und ,idr" „Herde“

\footnotetext{
${ }^{28}$ Grimal, La Stèle Triomphale de Pi(`Ankh)y au Musée du Caire JE 48862 et 47086 - 47089, Études sur la propaganda royale égyptienne, MIFAO 105 (1981), 53 Z. 32.

${ }^{29}$ Griffith/Thompson, The Demotic Magical Papyrus of London und Leiden, I. Text (1904), 177.

${ }^{30}$ Junker, Das Geburtshaus des Tempels der Isis in Philä (1965), 175.

${ }^{31}$ Lorand, CdE 83 (2008), 27/31.

${ }^{32}$ Das Wort „dnh" „Flügel“ hat bei Wreszinski, Der Londoner Medizinische Papyrus (Brit. Museum Nr. 10059) und der Papyrus Hearst in Transkription, Übersetzung und Kommentar (1912), 37, mit der Präposition , ,hn" „zusammen mit“ ein Wortspiel gebildet, welchem der bekannte Lautwandel zwischen ", und „ $d$ “" zugrunde liegt.

${ }_{33}$ Epigraphic Survey, The temple of Khonsu, Volume 2, Scenes and inscriptions in the court and the first hypostyle hall, OIP 103 (1981), 28/44.

${ }^{34}$ KRI IV, 297, 7.
} 
in: „iri $m i d r^{635}$ (NR), ,in Herden zusammenfassen“ benennen. Das Wortspiel zwischen „, $p 3 i^{\prime \prime}$ „fliegen“" und ,3pd“" „Vogel“ in: ,b3.w hpr.w $m$ 3pd.w p3i r p.t ${ }^{636}(\mathrm{NR})$,Bas werden zu Vögeln, die zum Himmel fliegen" ist ebenfalls durch diesen Übergang geprägt, wobei sich auch hier eine Metathese manifestiert. Die verderbte Schreibung, ,irnśs ${ }^{637}$

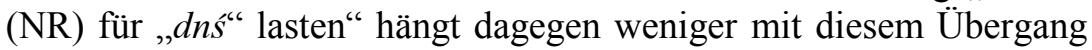
zusammen. Die Ursache dürfte vielmehr in der vagen Ähnlichkeit von 8 und im Hieratischen liegen.

\section{Der Übergang zwischen , ,i“ und,$\underline{d}^{\text {“‘ }}$}

Im vierten Abschnitt soll der Lautwandel zwischen , , $i^{\text {“ }}$ und , $\underline{d}^{\text {“6 }}$ thematisiert werden. Das Wortspiel zwischen „wi3“" „Barke“ und

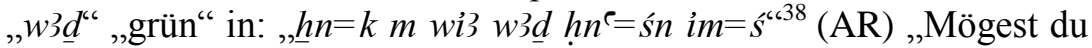
mit ihnen in der Grünen Barke rudern darin." soll dabei zuerst eingeführt werden. Die Begründung, dass hier wirklich ein Wortspiel unter Beteiligung dieses Übergangs vorliegt, wird weiter unten nachgeliefert. Die dortigen Indizien werden dafür eine umso deutlichere Sprache sprechen. Das Beispiel ist jedoch für die Geschichte dieses Übergangs von allergrößter Bedeutung, weil es zeigt, dass seine Anfänge bis ins Alte Reich zurückreichen. Das Wortspiel zwischen ,wij““ „Barke“ und ,w3 $\underline{d}^{\text {“ }}$,grün“ ist in: ,h $h i=k$

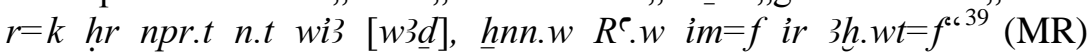
„Mögest du aber einsteigen auf dem Rand der [Grünen] Barke, mit der $\mathrm{Re}$ zu seinen Horizonten fährt." auch für das Mittlere Reich nachgewiesen. Die in einer Handschrift für , $m 3 w \underline{d}^{\text {“ }}{ }^{40}$ „Joch“

\footnotetext{
${ }^{35}$ Leitz, Tagewählerei, Das Buch h3.t nhh ph ph.wi d. $t$ und verwandte Texte, Textband, ÄgAb 55 (1994), 120.

${ }^{36}$ Leitz, Tagewählerei, Das Buch h3.t nhh ph.wi d.t und verwandte Texte, Textband ÄgAb 55 (1994), 39.

${ }^{37}$ Fischer-Elfert, Lesefunde im literarischen Steinbruch von Deir el-Medineh, KÄT 12 (1997), 56.

${ }^{38}$ Faulkner, The ancient Egyptian Pyramid Texts, Translated into English (1969), 279.

${ }^{39}$ Assmann, Altägyptische Totenliturgien, Band 1, Totenliturgien in den Sargtexten des Mittleren Reiches (2002), 361/457.

${ }^{40}$ Burkard, Textkritische Untersuchungen zu ägyptischen Weisheitslehren des Alten und Mittleren Reiches, ÄgAb 34 (1977), 54 zum „,m3wd“ - Stab vgl. auch Helck, Die Beziehungen Ägyptens zu Vorderasien im 3. und 2. Jahrtausend v. Chr., 2., verbesserte Auflage. ÄgAb 5 (1971), 513.
} 
gebrauchte Schreibung „m3ỉw“ (NR) „Löwe“ könnte ebenfalls durch diesen Übergang bedingt sein. In der Interpretation des Befundes hatte sich Burkard noch mit der Verlesung von $\bowtie$ in $\triangleq$ behelfen müssen. Das Wortspiel zwischen „wi ““ „Barke“ und „w3d“ ,grün“ ist mit

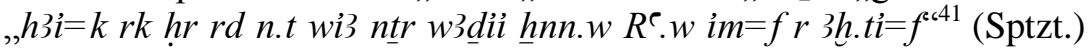
„Steige doch ein über den Rand der Grünen Gottesbarke, mit der Re zu seinen beiden Horizonten rudert.“ auch in Papyri der Spätzeit zu finden, die freilich in der Tradition älterer religiöser Texte stehen. Wie angekündigt, soll nun der Beweis erbracht werden, dass dieses Wortspiel nicht $\mathrm{zu}$ unrecht angenommen worden ist. In diesem Zusammenhang kann positiver Weise darauf hingewiesen werden,

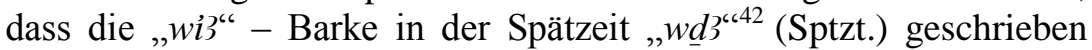
werden konnte. Das Argument dürfte ausreichen, um auch die letzten

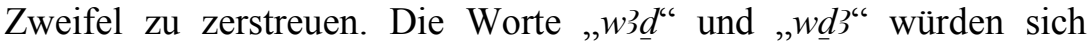
schließlich nur durch eine Metathese voneinander unterschieden. Die aus demotischen Urkunden bekannte Formel ,iri $\underline{d} r . t^{\star 43}$ (Sptzt.) „in Hand leisten" hat ebenfalls aus diesem Übergang ihre Kraft bezogen. Wenn die Ergänzung richtig ist, gehört auch das Wortspiel zwischen

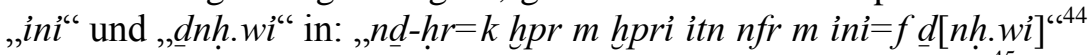
(Sptzt.) „Gegrüßt seiest du, der als Chepri entstanden ${ }^{45}$ ist, vollkommene ${ }^{46}$ Sonnenscheibe, wenn sie die [beiden] [Fl]ügel trägt"

\footnotetext{
${ }^{41}$ Assmann, Altägyptische Totenliturgien, 3. Band, Osirisliturgien in Papyri der Spätzeit (2008), 402.

${ }_{42}$ Kockelmann, Untersuchungen zu den späten Totenbuch-Handschriften auf Mumienbinden, Band I. 1, Die Mumienbinden und Leinenamulette des memphitischen Priesters Hor, SAT 12 (2008), 84.

${ }^{43}$ Kaplony-Heckel, Land und Leute am Nil nach demotischen Inschriften, Papyri und Ostraka, Gesammelte Schriften Teil 1, ÄgAb 71 (2009), 151.

${ }^{44}$ Pries, Das nächtliche Stundenritual zum Schutz des Königs und verwandte Kompositionen, Der Papyrus Kairo 58027 und die Textvarianten in den Geburtshäusern von Dendera und Edfu, SAGA 27 (2009), 110.

${ }^{45}$ Das gleiche Wortspiel kommt auch bei KRI II, 276, 5; Müller-Roth, Das Buch vom Tage, OBO 236 (2008), 106, vor.

${ }^{46}$ Das Attribut ,nfr" ist der , itn“" - Scheibe auch sonst verliehen worden, vgl. Urk. IV, 2095, 7; Urk. VIII, 44, 9; Urk. VIII, 45, 9; Urk. VIII, 63, 16; Chassinat, Le Temple de Dendara, Tome Deuxième, Publications de 1'Institut Francais d'Archéologie Orientale du Caire (1934), 35/167; Chassinat, Le Temple de Dendara, Tome Quatrième, Publications de 1'Institut Francais d'Archéologie Orientale du Caire (1935), 139; Kurth, Edfou VIII, Die Inschriften des Tempels von Edfu, Abteilung I, Übersetzungen Band 1 (1998), 173; Kurth, Edfou VII, Die Inschriften des Tempels von Edfu, Abteilung I, Übersetzungen Band 2 (2004), 108.
} 
hierher. Das Wortspiel wird außerdem durch die bekannte Verschreibung zwischen , "i“ und,$h^{\prime \prime}$ in seinem Innersten zusammen gehalten. Die Basis für die Schreibung ,śbi.w" „Rebellen“ anstelle

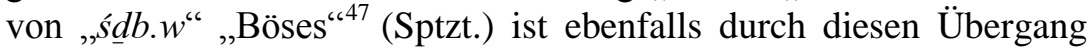
gelegt worden.

${ }^{47}$ Burkard, Spätzeitliche Osiris-Liturgien im Corpus der Asasif-Papyri, Übersetzung, Kommentar, Formale und inhaltliche Analyse, ÄAT 31 (1995), 182 n. 12. 
